\title{
Optimal Permanent-Magnet Geometries for Dipole Field Approximation
}

\author{
Andrew J. Petruska and Jake J. Abbott \\ Department of Mechanical Engineering, University of Utah, Salt Lake City, UT 84112 USA
}

\begin{abstract}
The dipole approximation for magnetic fields has become a common simplifying assumption in magnetic-manipulation research when dealing with permanent magnets because the approximation provides convenient analytical properties that are a good fit at large distances. What is meant by "good fit at large distances" is generally not quantified in the literature. By using a parameterized multipole expansion and collaborating finite-element analysis (FEA) simulations to represent the magnet's field, we quantify the error associated with the dipole approximation as a function of distance from the permanent magnet. Using this expression, we find cylindrical, washer, and rectangular-cross-section bar permanent-magnet aspect ratios that minimize the error of the dipole approximation. For cylinders and rectangular-cross-section bars, these aspect ratios are a diameter-to-length ratio of $\sqrt{4 / 3}$ and a cube, respectively.
\end{abstract}

Index Terms-Magnetic analysis, magnetostatics, permanent magnets.

\section{INTRODUCTION}

$\mathbf{T}$ HE magnetic fields generated by current distributions and permanent magnet can be modeled in numerous ways from direct integration of the Biot-Sivart law, to finite-element analysis (FEA), to harmonic expansions of the fields. The dipole approximation, the first spherical harmonic of the field, provides a concise and easily manipulated representation of the magnetic field and is increasingly accurate with distance. This approximation is commonly used for localization of objects in areas ranging from medical imaging applications [1], to military applications [2]-[4], to object tracking [5]-[20]. The dipole representation also provides interesting applications in real-time control of magnetic devices for medical applications [21]-[24]. A pure dipole field can be generated by a uniformly magnetized spherical permanent magnet; however, other shapes of permanent magnets can be represented by a dipole field as an approximation at large distances. Understanding the limitations of this approximation for the commonly available cylindrical, washer, and rectangular-cross-section bar permanent magnets is the focus of this paper, along with answering the question: "What shape of cylindrical/washer/bar permanent magnet is best represented by a pure dipole field?"

There are works that explicitly address the accuracy of the dipole approximations of permanent magnets. Hu et al. [6]-[9] propose placing a cylindrical permanent magnet inside a capsule endoscope for localization. They use the dipole approximation to describe the magnetic field produced by the permanent magnet and use linear calibration techniques to minimize the error associated with the strength of the magnet. Wang and Meng [11] explore the accuracy of the dipole model for two types of cylindrical magnets for use as magnetic markers in capsule endoscopes. They quantify the error along the axis and radius of the cylindrical magnet and suggest a rectangular "keep-out region" for the approximation that is three times the dimension of the magnet, but they do not examine the error associated with the model for other locations. They also indicate that

Manuscript received April 12, 2012; revised June 05, 2012; accepted June 07, 2012. Date of publication June 18, 2012; date of current version January 22, 2013. Corresponding author: A. J. Petruska (e-mail: Andrew.Petruska@utah. edu.).

Color versions of one or more of the figures in this paper are available online at http://ieeexplore.ieee.org.

Digital Object Identifier 10.1109/TMAG.2012.2205014 an axially magnetized magnet with a diameter-to-length ratio of 2.5 is better represented by the dipole model than an axially magnetized magnet with a diameter-to-length ratio of 0.5 . Fountain et al. [21] examine the use of a rotating permanent magnet to control helical swimmers and propose using a dipole model to represent the field of the cylindrical permanent magnets. The approximation is justified a posteriori by a least squares fit of the dipole model to experimental data collected along the magnetization axis for both diametrically magnetized and axially magnetized cylindrical magnets. Interestingly, the fit to the diametric magnet shows better agreement with the dipole model than the axial magnet with the same geometry. Mahoney et al. [23] continued this research and exploited the linear-algebraic properties of the dipole field to show interesting force and torque combinations can be applied by one rotating permanent magnet acting on another sympathetically rotating permanent magnet. They then use the dipole model to demonstrate that rotating magnetic fields can be generated about arbitrary axes in space using a single rotating permanent magnet in any relative position [24]. The above works show the dipole model is an accurate approximation for distances far away from the permanent magnet and imply that using a permanent magnet that is more accurately modeled by the dipole approximation at distances nearer to the magnet will enable more accurate real-time magnetic control of untethered devices. Two observations in the works by Fountain et al. and by Wang and Meng indicate there may be an optimal aspect ratio and magnetization direction to accurately represent the magnetic field produced by a cylindrical permanent magnet using the dipole approximation.

To explore the nature of a shape that is optimally represented by the dipole-model approximation and to quantify the error in the model at any location outside the magnet, a parameterized multipole expansion is presented in this paper for axially and diametrically magnetized cylindrical magnets and rectangular-cross-section bar magnets. The accuracy of this expansion is verified by comparisons to FEA models of the magnetic fields from different magnet geometries. Since the dipole approximation is the first term of this expansion, analytically calculating the optimal shape aspect ratios can be achieved by minimizing the contribution of the remaining terms to the field representation.

The paper is structured as follows. First, the theoretical foundation for a multipole expansion for representing the magnetic 
field of a permanent magnet is reviewed, and the criteria for defining the shape with minimal dipole-model error will be introduced. Then, the multipole expansion for axially and diametrically magnetized cylindrical magnets and rectangular-crosssection bar magnets is solved and compared to FEA models of several different magnet geometries to validate the expansion. The optimal aspect ratios for cylindrical magnets and rectangular bars is then solved and extended to washers. Finally, the error associated with the dipole approximation for both the optimal and other commonly available shapes is presented.

\section{Permanent-Magnet Field Approximation}

The magnetization $\mathbf{M}$ inside a permanent magnet is a function of the applied field $\mathbf{H}_{a}$, the magnetic remanence of the material $\mathbf{H}_{r}$, the susceptibility of the material $\chi$, and the shape demagnetization factors along the principal axes of the magnet $N_{i}$

$$
\mathbf{M}=\left[\begin{array}{ccc}
\frac{1}{1+\chi N_{1}} & 0 & 0 \\
0 & \frac{1}{1+\chi N_{2}} & 0 \\
0 & 0 & \frac{1}{1+\chi N_{3}}
\end{array}\right]\left(\chi \mathbf{H}_{a}+\mathbf{H}_{r}\right)
$$

where all vectors are expressed relative to the principal axes of the magnet. The demagnetization factors are a set of three fractional values, which sum to one, that describe how a given shape magnetically interacts with itself, and how susceptible the shape is to magnetization in each of the principle directions; for a sphere, the demagnetization factors are $1 / 3$ in each principle direction. The demagnetization factors for ellipsoids of revolution, cylinders with ellipsoidal cross sections, and rectangular bars have been calculated [25]-[28]. For hard-magnetic materials with relatively low magnetic recoil susceptibilities for applied fields weaker than the coercive field strength, such as $\mathrm{NdFeB}$ with $\chi \approx 0.05$, the demagnetizing field becomes negligible and the magnetization reduces to the magnetic remanence of the material. For a permanent magnet with no external field applied, the magnetic $\mathbf{H}$ field can be described as the gradient of a scalar potential $\Phi$, which can be defined by the magnetization of the object [29]

$$
\mathbf{H}=-\nabla \Phi, \quad \nabla^{2} \Phi=\nabla \cdot \mathbf{M} .
$$

This can be solved using direct integration [29]

$$
\Phi(\mathbf{p})=\frac{1}{4 \pi}\left(\int_{V} \frac{\nabla \cdot \mathbf{M}}{|\mathbf{p}-\boldsymbol{\rho}|} d v+\oint_{S} \frac{\hat{\mathbf{n}} \cdot \mathbf{M}}{|\mathbf{p}-\boldsymbol{\rho}|} d a\right)
$$

where $\mathbf{M}$ is the magnetization, $\hat{\mathbf{n}}$ is the normal unit vector pointing out of the surface of the magnetized material, $\mathbf{p}$ is the vector from the center of the magnetized volume to the point of interest and is independent of the integral, $\rho$ is the vector from the center of the magnetized volume to the point of integration, and $\hat{\mathbf{p}}$ and $\hat{\boldsymbol{\rho}}$ are their respective unit vectors.

For permanent magnets with low susceptibilities and uniform remanence, which is a reasonable assumption for permanent magnets that are saturated during manufacturing, the divergence in the volume $(\nabla \cdot \mathbf{M})$ is equal to zero, reducing (3) to

$$
\Phi(\mathbf{p})=\frac{1}{4 \pi} \oint_{S} \frac{\hat{\mathbf{n}} \cdot \mathbf{M}}{|\mathbf{p}-\boldsymbol{\rho}|} d a .
$$

This can be rewritten using a Taylor series as a multipole expansion if the points of interest are outside the minimum bounding sphere (i.e., the smallest sphere that will encompass the magnet) surrounding the permanent magnet [30]

$$
\Phi(\mathbf{p})=\frac{1}{4 \pi} \sum_{n=0}^{\infty} \frac{1}{p^{n+1}} \oint_{S} \rho^{n} P_{n}(\hat{\mathbf{p}} \cdot \hat{\boldsymbol{\rho}})(\hat{\mathbf{n}} \cdot \mathbf{M}) d a
$$

where $P_{n}()$ are Legendre polynomials $p=|\mathbf{p}|$ and $\rho=|\boldsymbol{\rho}|$. The magnetic field represented by this scalar potential is only defined outside the permanent magnet and is

$$
\mathbf{B}=\mu \mathbf{H}=-\mu \nabla \Phi
$$

where $\mu$ is the permeability of the surrounding medium (for free space $\mu=\mu_{0} \equiv 4 \pi \times 10^{-7} N \cdot A^{-2}$ ).

As a consequence of $\nabla \cdot \mathbf{B}=0$, no even terms (e.g., $\propto$ $\left.1 / p^{2}, \propto 1 / p^{4}, \ldots\right)$ exist in (6). The first nonzero term of the expansion is the dipole term $\left(\propto 1 / p^{3}\right)$, which is independent of geometry and is commonly used for approximation of the magnetic field for control applications because of its convenient vector form. This term is

$$
\mathbf{B}_{\text {dipole }}(\mathbf{p})=\frac{\mu_{0}}{4 \pi} \frac{1}{p^{3}}\left(3 \hat{\mathbf{p}} \hat{\mathbf{p}}^{\mathbf{T}}-\mathbf{I}\right) \mathbf{m}
$$

where $\mathbf{I}$ is a $3 \times 3$ identity matrix and $\mathbf{m}$ is being introduced here as the dipole moment of the object defined by

$$
\mathbf{m} \equiv \int_{V} \mathbf{M} d v .
$$

The next nonzero term in the series is the quadrupole term $(\propto$ $\left.1 / p^{5}\right)$ and the next is a hexapole $\left(\propto 1 / p^{7}\right)$, all of which are functions of magnet geometry. In these higher order terms, $\beta$ will represent any factors that parameterize the shape of the magnet, such as a diameter-to-length ratio for a cylinder.

For an approximation of the field consisting of the first $T$ terms, the relative error at any given point is

$$
\begin{aligned}
& \operatorname{Error}(\mathbf{p}, \beta) \\
& =\frac{\left|\mathbf{B}_{\text {actual }}-\mathbf{B}_{\text {approx }}\right|}{\left|\mathbf{B}_{\text {actual }}\right|} \\
& =\frac{\left|\mathbf{B}_{T+1}(\mathbf{p}, \beta)+\mathbf{B}_{T+2}(\mathbf{p}, \beta)+\sum_{n=T+3}^{\infty} \mathbf{B}_{n}(\mathbf{p}, \beta)\right|}{\left|\sum_{n=1}^{\infty} \mathbf{B}_{n}(\mathbf{p}, \beta)\right|} .
\end{aligned}
$$

Without loss of generality, $T$ will be assumed to be an odd number, requiring the even terms $\left(\mathbf{B}_{T+1}\right.$ and $\left.\mathbf{B}_{T+3}\right)$ to be zero, simplifying the error to

$$
\operatorname{Error}(\mathbf{p}, \beta)=\frac{\left|\mathbf{B}_{T+2}(\mathbf{p}, \beta)+\sum_{n=T+4}^{\infty} \mathbf{B}_{n}(\mathbf{p}, \beta)\right|}{\left|\sum_{n=1}^{\infty} \mathbf{B}_{n}(\mathbf{p}, \beta)\right|} .
$$




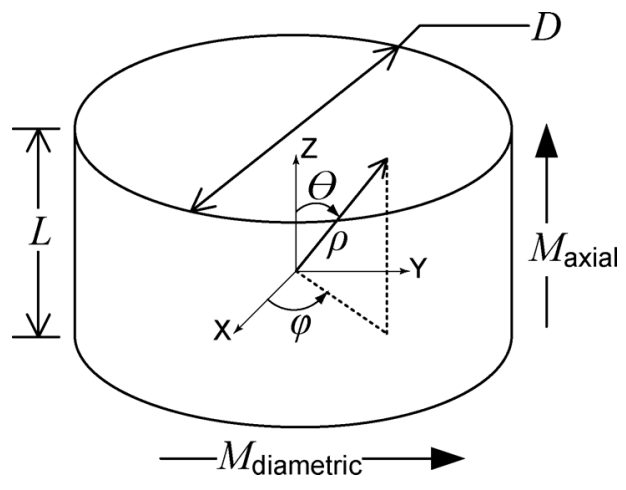

Fig. 1. Geometry definition for cylindrical magnets. The spherical coordinate definition is shown for the axially magnetized case only.

As $\mathbf{B}_{T+4}$ and higher order terms are asymptotically bounded by the $\mathbf{B}_{T+2}$ term, the optimal approximation geometry for distances much greater than the radius of the minimum bounding sphere radius $R_{s}$ can be determined by minimizing the magnitude of the $\mathbf{B}_{T+2}$ term alone. As this provides the optimal geometry for distances far away from the minimum bounding sphere, it is conceivable that at some intermediate distance a different geometry could provide a locally optimal solution. To determine locally optimal solutions, the integral of (10) over the region of interest would need to be minimized and more terms than just the $\mathbf{B}_{T+2}$ term would need to be considered. As this minimization is application specific, only the far-field optimal geometry for the dipole approximation $(T=1)$ will be considered in this paper and the quadrupole term $\mathbf{B}_{3}$ term will be minimized by finding the value of $\beta$ that sets the contribution of the $\mathbf{B}_{3}$ term to zero at every location in space.

\section{MAgnet Multipole EXPANSION}

\section{A. Cylindrical Magnet}

Cylindrical permanent magnets are most readily available in the axially magnetized and diametrically magnetized forms. Without loss of generality, the axis of the cylinder is aligned with the Cartesian $z$-axis, as shown in Fig. 1. The parameter that characterizes the shape of the cylinder is the diameter-to-length aspect ratio $\beta$. The following equations summarize some useful relationships using this parametrization, where $R_{s}$ is the radius of the minimum bounding sphere and $V$ is the volume of the cylinder:

$$
\begin{gathered}
\beta \equiv \frac{D}{L} \quad R_{s}=\left(\frac{V}{2 \pi}\right)^{\frac{1}{3}} \frac{\sqrt{\beta^{2}+1}}{\beta^{\frac{2}{3}}} \\
D=2\left(\frac{V \beta}{2 \pi}\right)^{\frac{1}{3}} \quad L=2\left(\frac{V}{2 \pi \beta^{2}}\right)^{\frac{1}{3}} .
\end{gathered}
$$

For materials like $\mathrm{NdFeB}$ with small susceptibilities $(|\chi| \ll 1)$, (1) and (8) simplify to $\mathbf{m} \approx \mathbf{H}_{r} V$, which, given a minimum bounding sphere, maximizes when $\beta=\sqrt{2}$. However, maximizing the dipole moment of the magnet given a sphere size does not ensure a dipole approximation with minimal error; for that, a multipole expansion of the shape is required.
In the following sections, a spherical coordinate system will be used. All primed variables are defined relative to the magnet for integration and all nonprimed coordinates are defined relative to a global coordinate system in which the point of interest $\mathbf{p}$ is defined. In this convention, $\theta^{\prime}$ is measured from the magnetization axis of the material, $\phi^{\prime}$ is measured from a convenient axis orthogonal to the magnetization axis (typically along the length of the magnet), and $\rho^{\prime}$ is defined as a radial distance from the center of the material. In the global frame, $\mathbf{p}$ is the vector from the center of the magnetized material to the point of interest and will be described in a spherical frame with: $\theta$ measured from the global $z$-axis and $\hat{\boldsymbol{\theta}}$ pointing in the positive $\theta$ direction, $\phi$ measured from the positive $x$-axis and $\hat{\phi}$ pointing in the positive $\phi$ direction, and $p$ taken as the magnitude of the $\mathbf{p}$ vector with $\hat{\mathbf{r}}$ pointing in the $\mathbf{p}$ direction (the difference in variable name is to avoid confusion when switching between spherical and coordinate-free descriptions). For reference, Fig. 1 shows the coordinate system with both the global and material coordinate systems aligned.

1) Axially Magnetized Cylinder: The multipole expansion defined by (5) is adapted to axially magnetized cylinders by taking $\hat{\mathbf{n}}^{\prime} \cdot \mathbf{M}$ to be $M \equiv\|\mathbf{M}\|$ on the top surface, $-M$ on the bottom surface, and 0 on the cylindrical wall

$$
\Phi(\mathbf{p}, \beta)=\frac{M}{4 \pi} \sum_{n=0}^{\infty} \frac{\left(\frac{L}{2}\right)^{n+2}}{p^{n+1}} D_{n}(\mathbf{p}, \beta)
$$

where

$$
\begin{aligned}
D_{n}(\mathbf{p}, \beta)= & \int_{0}^{2 \pi} \int_{0}^{\tan ^{-1}(\beta)} \frac{P_{n}\left(\hat{\mathbf{p}} \cdot \hat{\boldsymbol{\rho}}^{\prime}\right) \sin \theta^{\prime}}{\left(\cos \theta^{\prime}\right)^{n+3}} d \theta^{\prime} d \phi^{\prime} \\
& -(-1)^{n} \int_{0}^{2 \pi} \int_{\pi-\tan ^{-1}(\beta)}^{\pi} \frac{P_{n}\left(\hat{\mathbf{p}} \cdot \hat{\boldsymbol{\rho}}^{\prime}\right) \sin \theta^{\prime}}{\left(\cos \theta^{\prime}\right)^{n+3}} d \theta^{\prime} d \phi^{\prime} .
\end{aligned}
$$

Using the substitution $x=\cos \theta^{\prime}, D_{n}$ can be further simplified

$$
D_{n}(\mathbf{p}, \beta)=\left\{\begin{array}{cl}
2 \int_{\frac{1}{\sqrt{1+\beta^{2}}} \int_{0}^{2 \pi} \frac{P_{n}\left(\hat{\mathbf{p}} \cdot \hat{\boldsymbol{\rho}}^{\prime}\right)}{x^{n+3}} d \phi^{\prime} d x,} & n \text { odd } \\
0, & n \text { even }
\end{array}\right.
$$

where

$$
\hat{\mathbf{p}} \cdot \hat{\boldsymbol{\rho}}^{\prime}=\sqrt{1-x^{2}} \sin \theta\left(\sin \phi \sin \phi^{\prime}+\cos \phi \cos \phi^{\prime}\right)+x \cos \theta .
$$

The magnetic field of an axially magnetized cylindrical magnet described in cylindrical coordinates is then

$$
\mathbf{B}(\mathbf{p})=\frac{\mu_{0}}{4 \pi} \frac{m}{V} \sum_{n \text { odd }} \frac{\left(\frac{L}{2}\right)^{n+2}}{p^{n+2}}\left((n+1) D_{n} \hat{\mathbf{r}}-\frac{d D_{n}}{d \theta} \hat{\boldsymbol{\theta}}\right)
$$

where $m$ is the magnitude of the dipole moment $\mathbf{m}$. Noticing that $m \cos \theta \hat{\mathbf{r}}=\hat{\mathbf{p}} \hat{\mathbf{p}}^{\mathbf{T}} \mathbf{m}$ and that $m \sin \theta \hat{\boldsymbol{\theta}}=\left(\hat{\mathbf{p}} \hat{\mathbf{p}}^{\mathbf{T}}-\mathbf{I}\right) \mathbf{m}$, the spherical-coordinate field definition can be converted into a coordinate-free form. The first term is the dipole term, as expected. The next two terms will be used in Sections IV-A and IV-C to 
find the geometry that maximizes the contribution of the dipole term to total field

$$
\begin{aligned}
\mathbf{B}_{1}= & \frac{\mu_{0}}{4 \pi} \frac{1}{p^{3}}\left(3 \hat{\mathbf{p}} \hat{\mathbf{p}}^{\mathbf{T}}-\mathbf{I}\right) \mathbf{m} \\
\mathbf{B}_{3}= & \frac{\mu_{0}}{4 \pi} \frac{1}{p^{5}}\left(\frac{L}{2}\right)^{2}\left(\frac{4-3 \beta^{2}}{8}\right) \\
& \cdot\left(\left(35\left(\hat{\mathbf{m}}^{\mathbf{T}} \hat{\mathbf{p}}\right)^{2}-15\right) \hat{\mathbf{p}} \hat{\mathbf{p}}^{\mathbf{T}}-\left(15\left(\hat{\mathbf{m}}^{\mathbf{T}} \hat{\mathbf{p}}\right)^{2}-3\right) \mathbf{I}\right) \mathbf{m} \\
\mathbf{B}_{5}= & \frac{\mu_{0}}{4 \pi} \frac{1}{p^{7}}\left(\frac{L}{2}\right)^{4}\left(\frac{15 \beta^{4}-60 \beta^{2}+24}{64}\right) \\
& \cdot\left(\left(231\left(\hat{\mathbf{m}}^{\mathbf{T}} \hat{\mathbf{p}}\right)^{4}-\frac{105}{2}\left(\hat{\mathbf{m}}^{\mathbf{T}} \hat{\mathbf{p}}\right)^{2}+35\right) \hat{\mathbf{p}} \hat{\mathbf{p}}^{\mathbf{T}}\right. \\
& \left.\quad-\left(105\left(\hat{\mathbf{m}}^{\mathbf{T}} \hat{\mathbf{p}}\right)^{4}-70\left(\hat{\mathbf{m}}^{\mathbf{T}} \hat{\mathbf{p}}\right)^{2}+5\right) \mathbf{I}\right) \mathbf{m} .
\end{aligned}
$$

2) Diametrically Magnetized Cylinder: Adapting the multipole expansion to diametrically magnetized cylinders requires taking the $\hat{\mathbf{n}}^{\prime} \cdot \mathbf{M}$ term in (5) to be $M \sin \phi^{\prime}$ on the cylindrical wall and 0 on the top and bottom surfaces. This aligns the magnetization direction with the Cartesian $y$-axis, and the scalar potential described by (5) becomes

$$
\Phi(\mathbf{p}, \beta)=\frac{M}{4 \pi} \sum_{n=0}^{\infty} \frac{\left(\frac{D}{2}\right)^{n+2}}{p^{n+1}} D_{n}(\mathbf{p}, \beta)
$$

where

$$
D_{n}(\mathbf{p}, \beta)=\int_{\tan ^{-1} \beta}^{\pi-\tan ^{-1} \beta} \int_{0}^{2 \pi} \frac{P_{n}\left(\hat{\mathbf{p}} \cdot \hat{\boldsymbol{\rho}}^{\prime}\right) \sin \phi^{\prime}}{\left(\sin \theta^{\prime}\right)^{n+2}} d \phi^{\prime} d \theta^{\prime}
$$

and

$\hat{\mathbf{p}} \cdot \hat{\boldsymbol{\rho}}^{\prime}=\cos \theta \sin \theta^{\prime} \sin \phi^{\prime}+\cos \phi \sin \theta \cos \theta^{\prime}$

$$
+\sin \phi \sin \theta \sin \theta^{\prime} \cos \phi^{\prime} \text {. }
$$

The magnetic field described in spherical coordinates is then

$$
\begin{aligned}
\mathbf{B}(\mathbf{p})=\frac{\mu_{0}}{4 \pi} \frac{m}{V} \sum_{n \text { odd }} \frac{\left(\frac{D}{2}\right)^{n+2}}{p^{n+2}} & \left((n+1) D_{n} \hat{\mathbf{r}}\right. \\
& \left.-\frac{d D_{n}}{d \theta} \hat{\boldsymbol{\theta}}-\frac{1}{\sin \theta} \frac{d D_{n}}{d \phi} \hat{\boldsymbol{\phi}}\right) .
\end{aligned}
$$

Noticing that $m \cos \theta \hat{\mathbf{r}}=\hat{\mathbf{p}} \hat{\mathbf{p}}^{\mathbf{T}} \mathbf{m}, m \sin \theta \hat{\boldsymbol{\theta}}=\left(\hat{\mathbf{p}} \hat{\mathbf{p}}^{\mathbf{T}}-\mathbf{I}\right) \mathbf{m}$, and $m \sin \theta \hat{\boldsymbol{\phi}}=-\hat{\mathbf{p}} \times \mathbf{m}$, and defining $\hat{\mathbf{l}}$ to run along the axis of the magnet, the spherical-coordinate field definition can be converted into a coordinate-free form. The first term is the dipole term. The next two terms will be used in Sections IV-A and IV-C

$$
\begin{aligned}
\mathbf{B}_{1}= & \frac{\mu_{0}}{4 \pi} \frac{1}{p^{3}}\left(3 \hat{\mathbf{p}} \hat{\mathbf{p}}^{\mathbf{T}}-\mathbf{I}\right) \mathbf{m} \\
\mathbf{B}_{3}= & \frac{\mu_{0}}{4 \pi} \frac{1}{p^{5}}\left(\frac{D}{2}\right)^{2}\left(\frac{3 \beta^{2}-4}{8}\right) \\
& \cdot\left(\left(\left(35\left(\hat{\mathbf{m}}^{\mathbf{T}} \hat{\mathbf{p}}\right)^{2}-25\right)\left(\frac{\hat{\mathbf{l}}^{\mathbf{T}} \hat{\mathbf{p}}}{|\hat{\mathbf{p}} \times \hat{\mathbf{m}}|}\right)^{2}+5\right) \hat{\mathbf{p}} \hat{\mathbf{p}}^{\mathbf{T}} \mathbf{m}\right. \\
& \left(\left(15\left(\hat{\mathbf{m}}^{\mathbf{T}} \hat{\mathbf{p}}\right)^{2}-5\right)\left(\frac{\hat{\mathbf{l}}^{\mathbf{T}} \hat{\mathbf{p}}}{|\hat{\mathbf{p}} \times \hat{\mathbf{m}}|}\right)^{2}+1\right) \mathbf{m} \\
& \left.+10\left(\frac{\left(\hat{\mathbf{l}}^{\mathbf{T}} \hat{\mathbf{p}}\right)\left(\hat{\mathbf{m}}^{\mathbf{T}} \hat{\mathbf{p}}\right)\left(\hat{\mathbf{p}}^{\mathbf{T}}(\hat{\mathbf{m}} \times \hat{\mathbf{l}})\right)}{|\hat{\mathbf{p}} \times \hat{\mathbf{m}}|^{2}}\right) \hat{\mathbf{p}} \times \mathbf{m}\right)
\end{aligned}
$$

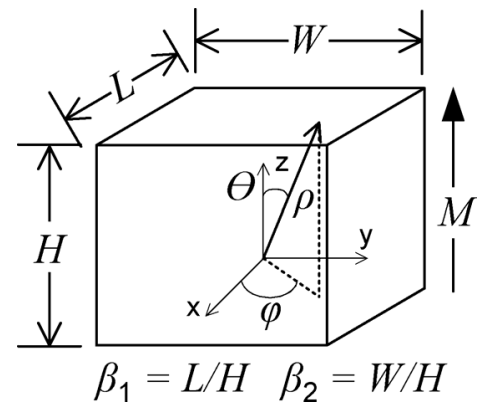

Fig. 2. Definition of a rectangular-cross-section bar magnet geometry.

$$
\begin{aligned}
& \mathbf{B}_{5}=\frac{\mu_{0}}{4 \pi} \frac{1}{p^{7}}\left(\frac{D}{2}\right)^{4}\left(\frac{5 \beta^{4}-20 \beta^{2}+8}{64}\right) \\
& \cdot\left(2 1 \left(3\left(7-11\left(\hat{\mathbf{m}}^{\mathbf{T}} \hat{\mathbf{p}}\right)^{2}\right) \frac{\left(\hat{\mathbf{l}}^{\mathbf{T}} \hat{\mathbf{p}}\right)^{4}}{|\hat{\mathbf{p}} \times \hat{\mathbf{m}}|^{2}}\right.\right. \\
&\left.+\left(18\left(\hat{\mathbf{m}}^{\mathbf{T}} \hat{\mathbf{p}}\right)^{2}-14\right)\left(\frac{\hat{\mathbf{l}}^{\mathbf{T}} \hat{\mathbf{p}}}{|\hat{\mathbf{p}} \times \hat{\mathbf{m}}|}\right)^{2}+1\right) \hat{\mathbf{p}} \hat{\mathbf{p}}^{\mathbf{T} \mathbf{m}} \\
&-3\left(21\left(1-5\left(\hat{\mathbf{m}}^{\mathbf{T}} \hat{\mathbf{p}}\right)^{2}\right) \frac{(\hat{\mathbf{l}} \mathbf{T} \hat{\mathbf{p}})^{4}}{|\hat{\mathbf{p}} \times \hat{\mathbf{m}}|^{2}}\right. \\
&\left.+\left(42\left(\hat{\mathbf{m}}^{\mathbf{T}} \hat{\mathbf{p}}\right)^{2}-14\right)\left(\frac{\hat{\mathbf{l}}^{\mathbf{T}} \hat{\mathbf{p}}}{|\hat{\mathbf{p}} \times \hat{\mathbf{m}}|}\right)^{2}+1\right) \mathbf{m} \\
&\left.-84 \frac{\hat{\mathbf{l}}^{\mathbf{T}} \hat{\mathbf{p}} \hat{\mathbf{m}}^{\mathbf{T}} \hat{\mathbf{p}} \hat{\mathbf{p}}^{\mathbf{T}}(\hat{\mathbf{m}} \times \hat{\mathbf{l}})}{|\hat{\mathbf{p}} \times \hat{\mathbf{m}}|^{2}}\left(3\left(\hat{\mathbf{l}}^{\mathbf{T}} \hat{\mathbf{p}}\right)^{2}-1\right) \hat{\mathbf{p}} \times \mathbf{m}\right) .
\end{aligned}
$$

\section{B. Rectangular-Cross-Section Bar Magnet}

Rectangular bar magnets require two aspect ratios to describe their shape. For this discussion, the height of the magnet $H$ will be taken along the magnetization direction and will be oriented with the $z$-axis, and $\beta_{1}$ and $\beta_{2}$ will be taken as the length-toheight and width-to-height aspect ratios and will be oriented along the $x$ - and $y$-axes, respectively. The geometry is shown in Fig. 2. For this geometry, (5) is adapted by taking $\hat{\mathbf{n}}^{\prime} \cdot \mathbf{M}$ to be $+M$ on the top and $-M$ on the bottom and becomes

$$
\Phi(\mathbf{p})=\frac{M}{4 \pi} \sum_{n=0}^{\infty} \frac{D_{n}\left(\mathbf{p}, \beta_{1}, \beta_{2}\right)}{p^{n+1}}
$$

where

$$
\begin{aligned}
D_{n}\left(\mathbf{p}, \beta_{1}, \beta_{2}\right) & =\left.\int_{-\beta_{2} H / 2}^{\frac{\beta_{2} H}{2}} \int_{-\beta_{1} H / 2}^{\frac{\beta_{1} H}{2}}\left(\rho^{\prime}\right)^{n} P_{n}\left(\hat{\mathbf{p}} \cdot \hat{\boldsymbol{\rho}}^{\prime}\right) d x^{\prime} d y^{\prime}\right|_{z^{\prime}=\frac{H}{2}} \\
& -\left.\int_{-\beta_{2} H / 2}^{\frac{\beta_{2} H}{2}} \int_{-\beta_{1} H / 2}^{\frac{\beta_{1} H}{2}}\left(\rho^{\prime}\right)^{n} P_{n}\left(\hat{\mathbf{p}} \cdot \hat{\boldsymbol{\rho}}^{\prime}\right) d x^{\prime} d y^{\prime}\right|_{z^{\circ}=-\frac{H}{2}}
\end{aligned}
$$

and

$$
\hat{\mathbf{p}} \cdot \hat{\boldsymbol{\rho}}^{\prime}=\frac{x^{\prime} \cos \phi \sin \theta+y^{\prime} \sin \phi \sin \theta+z^{\prime} \cos \theta}{\sqrt{x^{\prime 2}+y^{\prime 2}+z^{\prime 2}}} .
$$


$D_{n}$ can be further simplified to

$$
\begin{aligned}
& D_{n}\left(\mathbf{p}, \beta_{1}, \beta_{2}\right) \\
& =\left\{\begin{array}{cc}
\left.2 \int_{-\beta_{2} H / 2}^{\frac{\beta_{2} H}{2}} \int_{-\beta_{1} H / 2}^{\frac{\beta_{1} H}{2}}\left(\rho^{\prime}\right)^{n} P_{n}\left(\hat{\mathbf{p}} \cdot \hat{\boldsymbol{\rho}}^{\prime}\right) d x^{\prime} d y^{\prime}\right|_{z=\frac{H}{2}}, & n \text { odd } \\
0, & n \text { even. }
\end{array}\right.
\end{aligned}
$$

The magnetic field described in spherical coordinates is then

$$
\mathbf{B}(\mathbf{p})=\frac{\mu_{0}}{4 \pi} \frac{m}{V} \sum_{\mathrm{n} \text { odd }} \frac{1}{p^{n+2}}\left((n+1) D_{n} \hat{\mathbf{r}}-\frac{d D_{n}}{d \theta} \hat{\boldsymbol{\theta}}-\frac{1}{\sin \theta} \frac{d D_{n}}{d \phi} \hat{\boldsymbol{\phi}}\right) .
$$

Defining $\hat{\mathbf{l}}$ to point along the $L$ direction, and noticing that $m \cos \theta \hat{\mathbf{r}}=\hat{\mathbf{p}} \hat{\mathbf{p}}^{\mathbf{T}} \mathbf{m}, m \sin \theta \hat{\boldsymbol{\theta}}=\left(\hat{\mathbf{p}} \hat{\mathbf{p}}^{\mathbf{T}}-\mathbf{I}\right) \mathbf{m}$, $m \sin \theta \hat{\boldsymbol{\phi}}=-\hat{\mathbf{p}} \times \mathbf{m}, \cos \phi=\hat{\mathbf{p}}^{\mathbf{T}} \hat{\mathbf{l}} /|\hat{\mathbf{p}} \times \hat{\mathbf{m}}|$, and $\sin \phi=$ $\hat{\mathbf{p}}^{\mathbf{T}}(\hat{\mathbf{m}} \times \hat{\mathbf{1}}) /|\hat{\mathbf{p}} \times \hat{\mathbf{m}}|$, the spherical-coordinate field definition can be converted into a coordinate-free form. The first term is the dipole term. The next term will be used in Section IV-B

$$
\begin{aligned}
\mathbf{B}_{1}=\frac{\mu_{0}}{4 \pi} \frac{1}{p^{3}}\left(3 \hat{\mathbf{p}} \hat{\mathbf{p}}^{\mathbf{T}}-\mathbf{I}\right) \mathbf{m} & \mathbf{B}_{3}=\frac{\mu_{0}}{4 \pi} \frac{H^{2}}{8 p^{5}}\left(5 \left(\left(\beta_{1}^{2}-\beta_{2}^{2}\right)\left(7\left(\hat{\mathbf{m}}^{\mathbf{T}} \hat{\mathbf{p}}\right)^{2}+5\right)\left(\frac{\hat{\mathbf{p}}^{\mathbf{T}} \hat{\mathbf{l}}}{|\hat{\mathbf{p}} \times \hat{\mathbf{m}}|}\right)^{2}\right.\right. \\
& \left.-7\left(\beta_{1}^{2}-1\right)\left(\hat{\mathbf{m}}^{\mathbf{T}} \hat{\mathbf{p}}\right)^{2}-\beta_{1}^{2}+4 \beta_{2}^{2}-3\right) \hat{\mathbf{p}} \hat{\mathbf{p}}^{\mathbf{T}} \mathbf{m} \\
+ & \left(5\left(\beta_{1}^{2}-\beta_{2}^{2}\right)\left(\frac{\hat{\mathbf{p}}^{\mathbf{T}} \hat{\mathbf{l}}}{|\hat{\mathbf{p}} \times \hat{\mathbf{m}}|}\right)^{2}\left(3\left(\hat{\mathbf{m}}^{\mathbf{T}} \hat{\mathbf{p}}\right)^{2} 1\right)\right. \\
& \left.+15\left(\beta_{2}^{2}-1\right)\left(\hat{\mathbf{m}}^{\mathbf{T}} \hat{\mathbf{p}}\right)^{2}+\beta_{1}^{2}-4 \beta_{2}^{2}+3\right) \mathbf{m} \\
& +\frac{5}{2}\left(\beta_{2}^{2}-\beta_{1}^{2}\right) \frac{\left(\hat{\mathbf{p}}^{\mathbf{T}} \hat{\mathbf{l}}\right)\left(\hat{\mathbf{p}}^{\mathbf{T}}(\hat{\mathbf{m}} \times \hat{\mathbf{l}})\right)}{|\hat{\mathbf{p}} \times \hat{\mathbf{m}}|^{2}} \\
& \left.\times\left(\hat{\mathbf{m}}^{\mathbf{T}} \hat{\mathbf{p}}\right) \hat{\mathbf{p}} \times \mathbf{m}\right) .
\end{aligned}
$$

\section{Validation of Multipole Expansions With FEA Solutions}

To verify the validity of the expansions, the first nine nonzero terms of the multipole expansions are compared to FEA simulations solved by Ansoft Maxwell version 14.0. A cross-sectional contour plot showing the relative error between the multipole expansion and the FEA simulation for axially and diametrically magnetized cylinders and rectangular-cross-section bars are shown in Fig. 3. The average and maximum errors at a given distance are shown in Fig. 4. These comparisons demonstrate that the first nine terms of the multipole model are quite accurate for distances greater than 1.5 minimum-bounding-sphere radii.

\section{Optimal GeOMETRIC RELATIONS FOR DiPOLE APPROXIMATION}

\section{A. Cylinders}

In both the axially magnetized and diametrically magnetized conditions, the cylindrical magnet has the same shape-dependent factors in the multipole expansion, as shown by the second
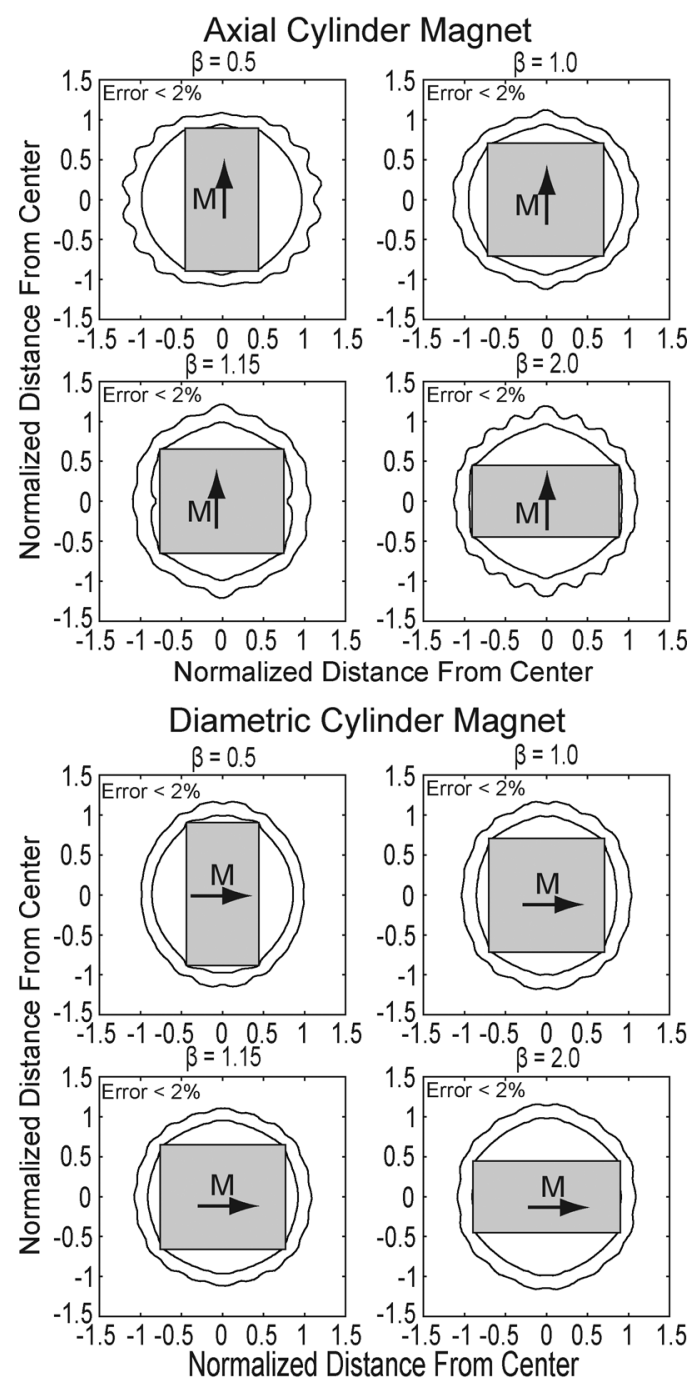

Rectangular Bar Magnet

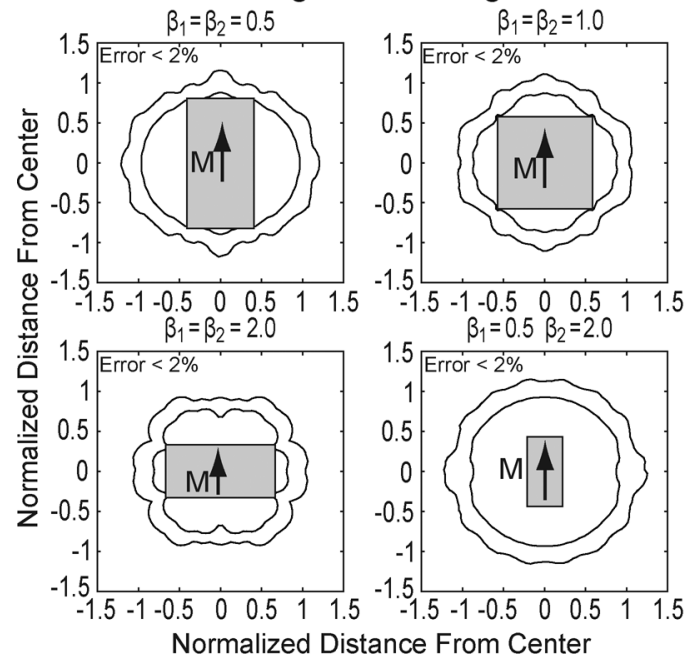

Fig. 3. Contour plot showing the $2 \%$ (outer) and $50 \%$ (inner) bands of error between the multipole expansion and the FEA model for several geometries of cylinder and rectangular magnets. Note that the error drops to below $2 \%$ after 1.5 radii of the minimum bounding sphere for each.

terms in (15) and (18), respectively. Using the far-field criterion for an optimal approximation geometry, the optimal $\beta$ is defined by the equation $4-3 \beta^{2}=0$. Therefore, the far-field optimal 

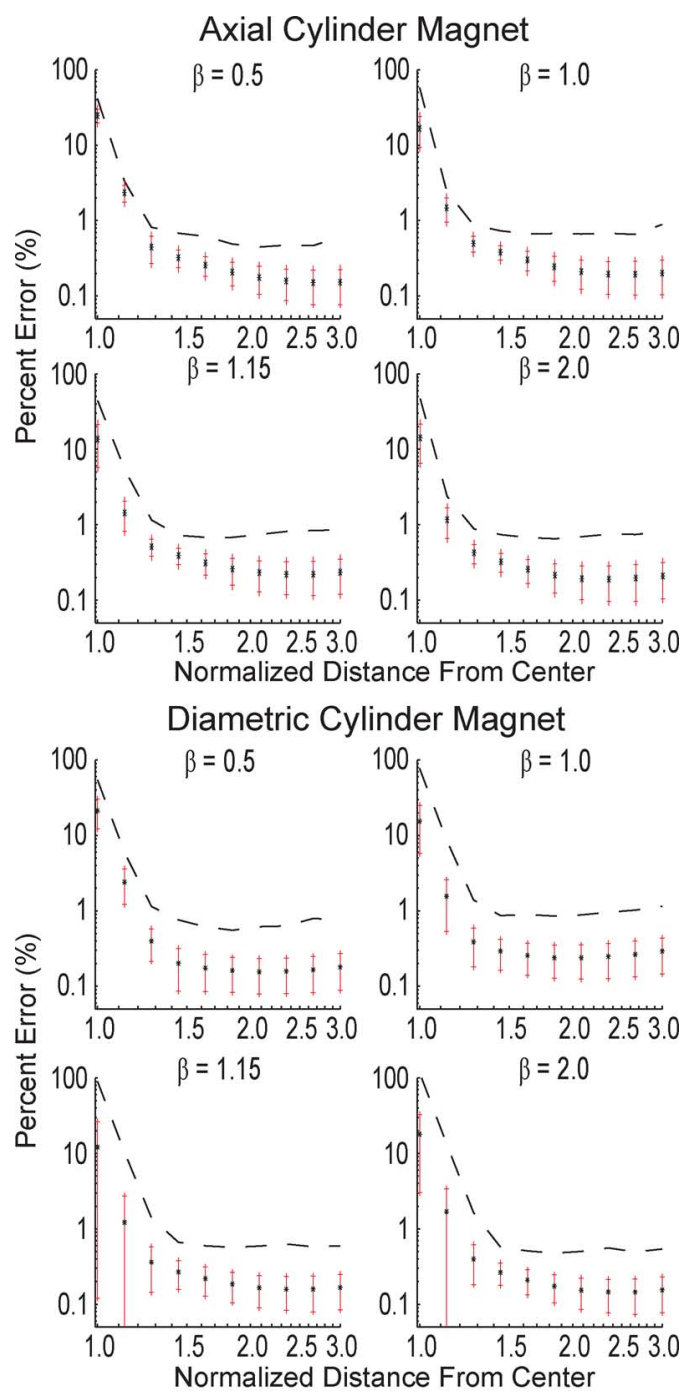

Rectangular Bar Magnet

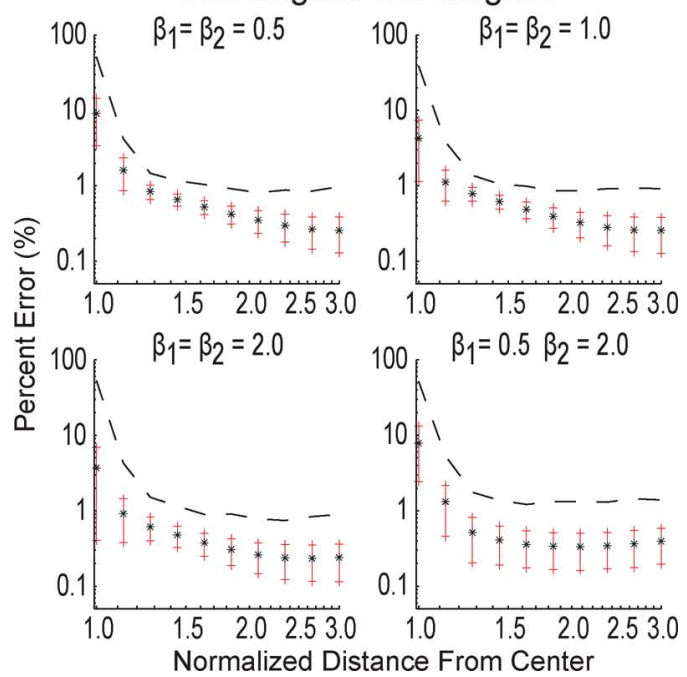

Fig. 4. The average error of the first nine terms of the multipole approximation as compared to FEA simulation over a spherical surface as a function of distance for several different geometries of cylinder and rectangular magnets. The dashed line represents the maximum error and the error bands \pm 1 standard deviation about the average error.

$\beta$ for both the diametrically magnetized and axially magnetized conditions is $\beta=\sqrt{4 / 3} \approx 1.15$. This aspect ratio corresponds to a $3 \%$ compromise in magnet volume and, therefore, dipole moment from the maximum given a minimum bounding sphere, which occurs when $\beta=\sqrt{2}$.

\section{B. Rectangular-Cross-Section Bar}

The optimal dipole approximation for a rectangular-crosssection bar can be obtained by determining which values of $\beta_{1}$ and $\beta_{2}$ set the $\mathbf{B}_{3}$ term in the multipole expansion defined in (21) to zero. Inspection of (21) shows that $\beta_{1}=\beta_{2}=1$ is the only solution that sets every component of the $\mathbf{B}_{3}$ coefficient to zero. Conveniently, this cubic geometry also corresponds to the maximum dipole moment for a given minimum bounding sphere.

\section{Axially Magnetized Washer}

As superposition holds for permanent magnet materials with low recoil susceptibilities (i.e., an externally applied or self-generated field does not appreciable affect the magnetization of the material), the optimal dipole shape for the washer shown in Fig. 5 can be defined by a linear combination of two cylinders of equal length. The larger diameter cylinder is taken to have a magnetization of $\mathbf{M}$ and a diameter-to-length ratio of $\beta_{1}$ and the smaller is taken to have a magnetization of $-\mathbf{M}$ and a diameter-to-length ratio of $\beta_{2}$. The volumes that these two cylinders overlap is equivalent to a hole in the larger cylinder. Following the procedure outlined previously, the equation that defines the optimal dipole approximation geometry for an axially magnetized washer is a linear combination of the $\mathbf{B}_{3}$ coefficients from (15) of the two cylinders scaled by their volumes $\left(V_{1}\right.$ and $\left.V_{2}\right)$

$$
V_{1}\left(\frac{L}{2}\right)^{2} \frac{4-3 \beta_{1}^{2}}{8}-V_{2}\left(\frac{L}{2}\right)^{2} \frac{4-3 \beta_{2}^{2}}{8}=0 .
$$

There is a real solution when $\sqrt{2 / 3}<\beta_{1} \leq \sqrt{4 / 3}$

$$
\beta_{2}=\frac{\sqrt{12-9 \beta_{1}}}{3} .
$$

Outside this range, the optimal dipole approximation is defined by minimizing the square of the quadrupole coefficient and is equivalent to having no hole or no magnet. Moreover, the only real values of $\beta_{1}$ and $\beta_{2}$ subject to (23) that minimize the hexapole $\mathbf{B}_{5}$ coefficient are $\sqrt{4 / 3}$ and 0 , respectively. That is, adding a hole to a nonoptimal configuration can make the approximation better, but the best geometry for dipole approximation has no hole. If a hole is desired, the optimal washer length can be calculated by substituting the definitions of $\beta_{1}$ and $\beta_{2}$ into (23) and solving for $L$, and is

$$
L=\sqrt{\frac{3}{4}\left(D_{1}^{2}+D_{2}^{2}\right)} .
$$

\section{Diametrically Magnetized Washer}

For a diametrically magnetized washer, the optimal hole size is defined by a linear combination of the $\mathbf{B}_{3}$ coefficients from (18) of the two cylinders scaled by their volume

$$
V_{1}\left(\frac{D_{1}}{2}\right)^{2} \frac{3 \beta_{1}^{2}-4}{8}-V_{2}\left(\frac{D_{2}}{2}\right)^{2} \frac{3 \beta_{2}^{2}-4}{8}=0
$$




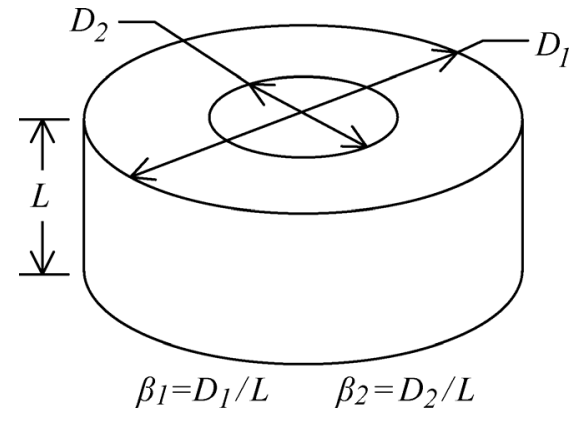

Fig. 5. Definition of washer-shaped magnet geometry.

which exists when $(2 \sqrt{2} / 3)<\beta_{1}<\sqrt{4 / 3}$ and is equal to

$$
\beta_{2}=\sqrt{\frac{1}{6}\left(\sqrt{\left(4-3 \beta_{1}^{2}\right)\left(9 \beta_{1}^{2}+4\right)}+4-3 \beta_{1}^{2}\right)} .
$$

Using this equation to define the relationship between $\beta_{1}$ and $\beta_{2}$, the magnitude of the hexapole term in (18) can be minimized

$$
\left|V_{1}\left(\frac{D_{1}}{2}\right)^{4} \frac{5 \beta_{1}^{4}-20 \beta_{1}^{2}+8}{64}-V_{2}\left(\frac{D_{2}}{2}\right)^{4} \frac{5 \beta_{2}^{4}-20 \beta_{2}^{2}+8}{64}\right| \rightarrow 0
$$

which minimizes when $\beta_{1}=\beta_{2}=2 \sqrt{2} / 3$ or, equivalently, the no-magnet geometry (i.e., the hole is the same size as the magnet). If (27) is normalized by the remaining dipole moment $\left(\|\mathbf{M}\|\left(V_{1}-V_{2}\right)\right)$, the resulting formula minimizes at the no-hole geometry; that is, the optimal geometry for a diametrically magnetized cylindrical magnet has no hole. If a hole is desired for a diametrically magnetized magnet, the optimal length is

$$
L=\sqrt{\frac{3}{4}\left(\frac{D_{1}^{4}+D_{1}^{2} D_{2}^{2}+D_{2}^{4}}{D_{1}^{2}+D_{2}^{2}}\right)} .
$$

\section{ERror Characterization of Optimal Dipole GEOMETRIES}

The average percent error over the surface of a sphere associated with the dipole approximation can be quantified by averaging (10) over the surface of a sphere (and multiplying by 100). The average error as a function of distance from the center of the magnet is given for axially magnetized cylinders, diametrically magnetized cylinders, and rectangular-cross-section rods in Fig. 6.

The crossover between the $\beta=1$ and $\beta=\sqrt{4 / 3}$ error curves, shown in the diametrically magnetized cylindrical magnet error plot, indicate that a geometry other than the far-field optimal will minimize the dipole approximation error for distances close to the surface of the magnet. Numerical analysis of diametrically magnetized cylinder magnets indicates that $\beta=1.10$ minimizes the dipole approximation error for distances between 1 and 4 minimum-bounding-sphere radii; however, the optimal near-field geometry provides only a marginal reduction in error when compared to the far-field optimal geometry at those distances.
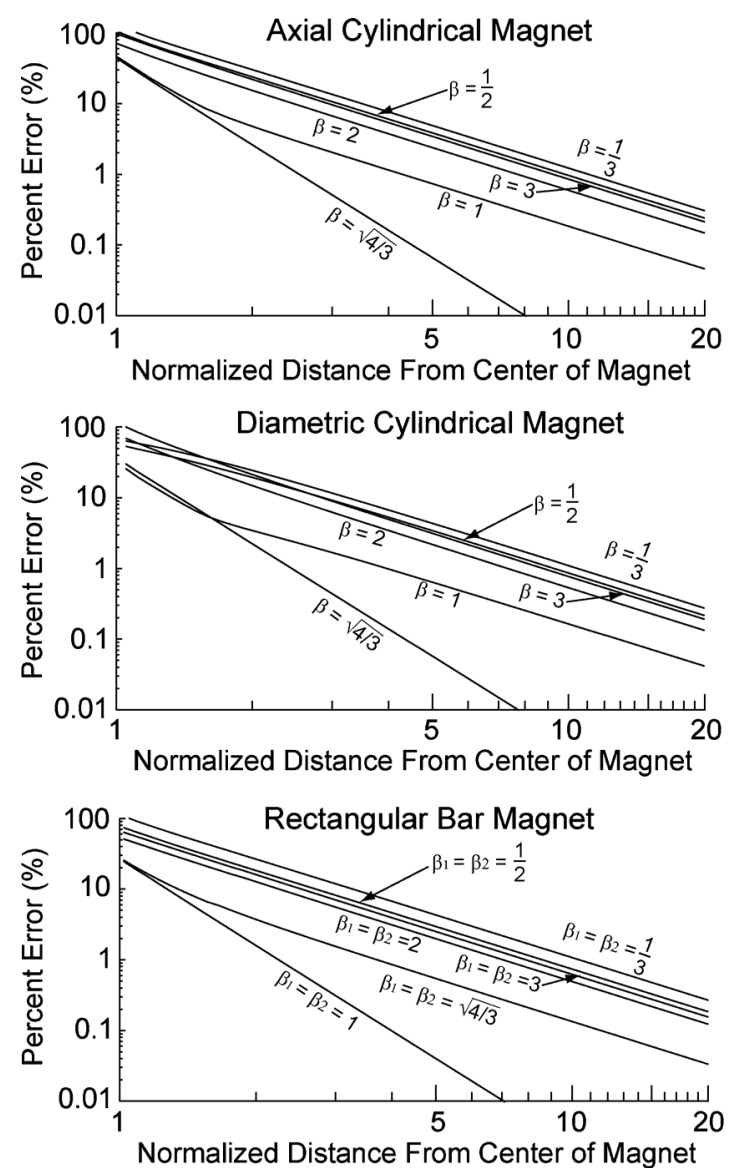

Fig. 6. The average errors associated with different geometries of cylindrical and rectangular permanent magnets are plotted as a function of distance from the center of the magnet. Distances are normalized by the radius of the minimum bounding sphere.

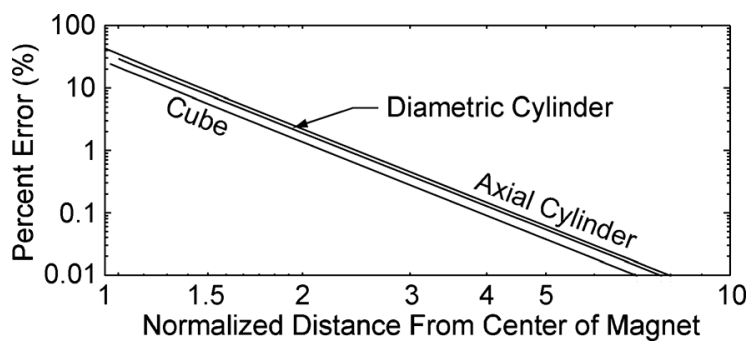

Fig. 7. The error associated with the optimal dipole geometries. The cubeshaped magnet has the least dipole approximation error, followed by the diametrically magnetized cylinder.

A comparison of the average error associated with the optimal geometries for the cylindrical and rectangular-cross-section bar magnets is shown in Fig. 7. The average error associated with the different optimal geometries is very close, with the cubic magnet having the least and the axially magnetized magnet having the most. Figs. 8 and 9 show the variation of error as a function of angle at a given distance. To determine the error at a given position, it is only necessary to multiply the average error given in Fig. 7 by the value in the error variation plot that corresponds to the angular position. 


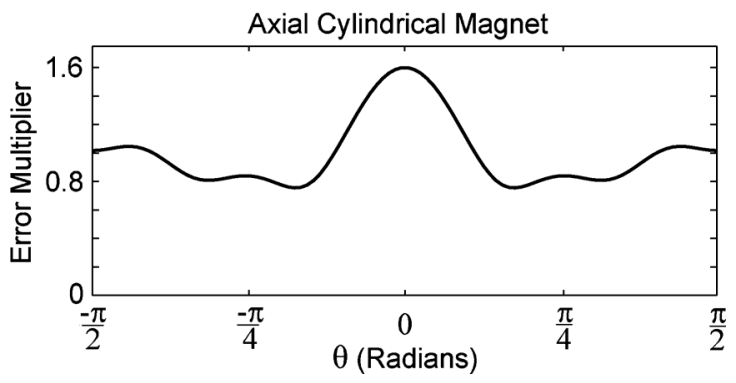

Fig. 8. Variation in dipole approximation error as a function of angle for an axially magnetized cylindrical magnet with $\beta=\sqrt{4 / 3}$. The magnetization axis of the magnet corresponds to $\theta=0$. See Fig. 1 .

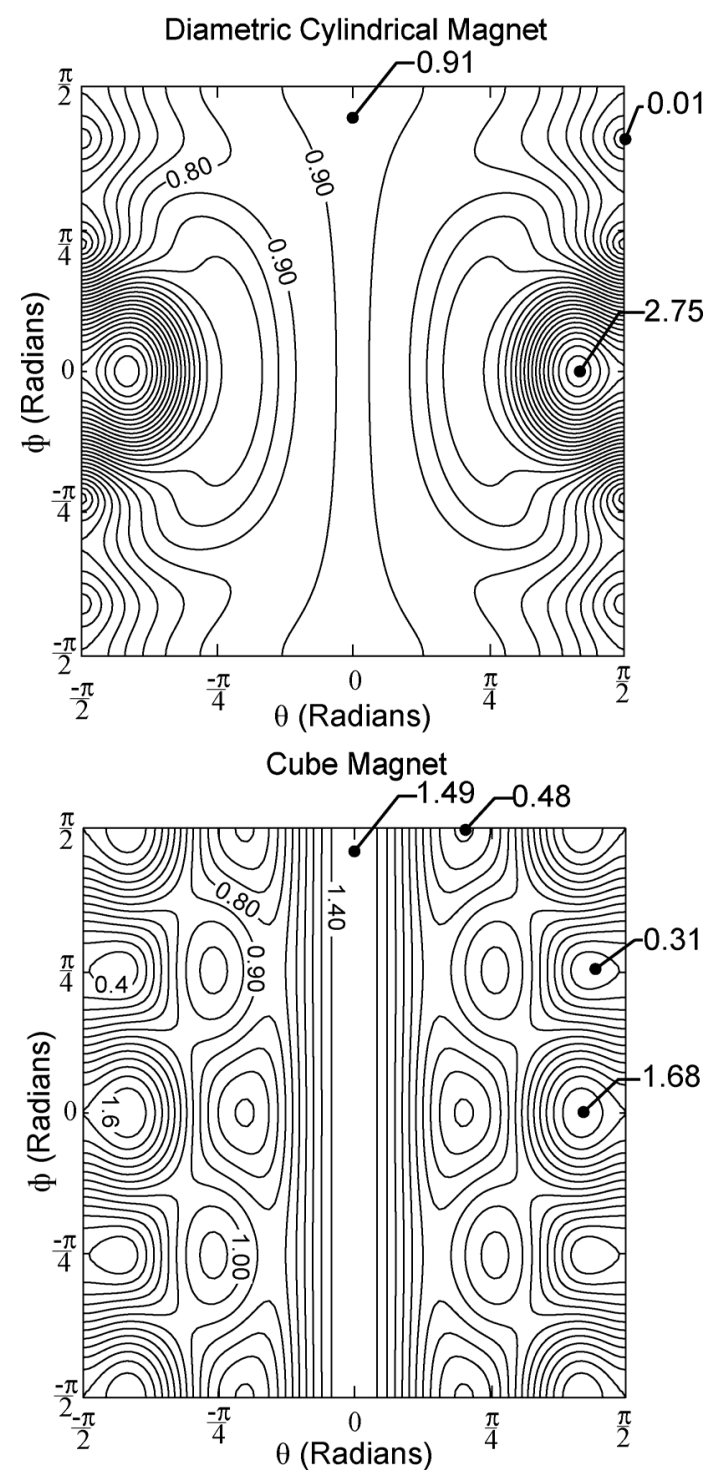

Fig. 9. Variation in dipole approximation error as a function of angle for an optimal diametrically magnetized cylindrical magnet and cube magnet. Each contour line represents a step in 0.1 times the average error at a given radius. The magnetization axis corresponds to $\theta=0$ and the $\hat{\mathbf{I}}$ direction corresponds to $\theta=\pi / 2$ and $\phi=0$. See Figs. 1 and 2 .

The diametrically magnetized cylinder has both the highest average error and the largest error range, which appears in conflict with the observations in the work by Fountain et al. [21], which shows diametrically magnetized magnets are preferable to the axially magnetized magnets. However, upon closer inspection, the least squares fit of the dipole approximation in the work by Fountain et al. is based on field measurements taken only along the magnetization axis of the magnet. At those locations, Figs. 7-9 predict a lower error for diametrically magnetized magnets than axially magnetized magnets, since the average error is approximately the same and the error multiplier for $\theta=0$ is 0.91 versus 1.6, respectively. Because Fountain et al. took their data along the magnetization axis, the dipole fit not only was better for the diametrically magnetized magnets, but also was more accurate in determining the dipole moment of the magnet. This also explains why Fountain et al. calculated different dipole moments based on their measured data for the axially magnetized magnet and the diametrically magnetized magnet despite both magnets having the same volume and material.

\section{CONCLUSION}

The multipole expansion provides an accurate method for determining the field generated by a permanent magnet for positions away from the surface of the magnet. This expansion also provides a direct way to determine the optimal dipole approximation geometric parameters for various shapes of magnets. This optimal geometry for cylinders (both axially and diametrically magnetized) is a diameter-to-length ratio of $\sqrt{4 / 3}$ and, for rectangular-cross-section bars, it is a cube. By choosing these ratios, the error associated with the dipole model is reduced compared to nonoptimal geometries, as shown by the trend depicted in Fig. 6. The accuracy of the approximation increases faster with distance for optimal geometries than it does for nonoptimal geometries. Given a minimum bounding sphere, there is no reduction in dipole moment magnitude from the maximum in rectangular bars to achieve an optimal dipole approximation and the reduction required in cylinders is less than $3 \%$. Of the geometries studied for a given distance from the magnet, the cubic magnet has the least average dipole approximation error followed closely by the diametric and axial magnets. The axially magnetized cylindrical magnet has the smallest range in error at a given distance from the center of the magnet.

\section{ACKNOWLEDGMENT}

This work was supported by the National Science Foundation under Grants 0952718 and 0654414.

\section{REFERENCES}

[1] M. Hämäläinen, R. Hari, R. J. Ilmoniemi, J. Knuutila, and O. V. Lounasmaa, "Magnetoencephalography-Theory, instrumentation, and applications to noninvasive studies of the working human brain," Rev. Mod. Phys., vol. 65, pp. 413-497, 1993.

[2] F. Raab, E. Blood, T. Steiner, and H. Jones, "Magnetic position and orientation tracking system," IEEE Trans. Aerosp. Electron. Syst., vol. AES-15, no. 5, pp. 709-718, Sep. 1979.

[3] A. Sheinker, L. Frumkis, B. Ginzburg, N. Salomonski, and B.-Z. Kaplan, "Magnetic anomaly detection using a three-axis magnetometer," IEEE Trans. Magn., vol. 45, no. 1, pp. 160-167, Jan. 2009.

[4] M. Birsan, "Recursive Bayesian method for magnetic dipole tracking with a tensor gradiometer," IEEE Trans. Magn., vol. 47, no. 2, pp. 409-415, Feb. 2011. 
[5] E. Paperno, I. Sasada, and E. Leonovich, "A new method for magnetic position and orientation tracking," IEEE Trans. Magn., vol. 37, no. 4, pp. 1938-1940, Jul. 2001.

[6] C. Hu, M. Q. Meng, and M. Mandal, "Efficient magnetic localization and orientation technique for capsule endoscopy," in Proc. IEEE/RSJ Int. Conf. Intell. Robots Syst., 2005, pp. 628-633.

[7] C. Hu, M. Q. Meng, and M. Mandal, "The calibration of 3-axis magnetic sensor array system for tracking wireless capsule endoscope," in Proc. IEEE/RSJ Int. Conf. Intell. Robots Syst., Oct. 2006, pp. $162-167$.

[8] C. Hu, M. Q. Meng, and M. Mandal, "A linear algorithm for tracing magnet position and orientation by using three-axis magnetic sensors," IEEE Trans. Magn., vol. 43, no. 12, pp. 4096-4101, Dec. 2007.

[9] C. Hu, M. Li, S. Song, W. Yang, R. Zhang, and M.-H. Meng, "A cubic 3-axis magnetic sensor array for wirelessly tracking magnet position and orientation," IEEE Sensors, vol. 10, no. 5, pp. 903-913, May 2010.

[10] C. Hu, S. Song, X. Wang, M. Meng, and B. Li, "A novel positioning and orientation system based on 3-axis magnetic coils," IEEE Trans. Magn., 2012, to be published.

[11] X. Wang and M. Meng, "Dipole modeling of magnetic marker for capsule endoscope localization," in Proc. World Congr. Intell. Control Autom., 2006, vol. 2, pp. 5382-5386.

[12] X. Wang and M. Q.-H. Meng, "Application of a magnetic dipole modelling approach to the problem of tracking a capsule endoscope," Proc. Inst. Mech. Eng. H/J. Eng. Med., vol. 225, no. 4, pp. 377-398, Apr. 2011.

[13] J. Baldoni and B. Yellen, "Magnetic tracking system: Monitoring heart valve prostheses," IEEE Trans. Magn., vol. 43, no. 6, pp. 2430-2432, Jun. 2007.

[14] S. Song, C. Hu, M. Li, W. Yang, and M.-H. Meng, "Two-magnet-based 6d-localization and orientation for wireless capsule endoscope," in Proc. IEEE Int. Conf. Robot. Biomimetics, Dec. 2009, pp. 2338-2343.

[15] G. Ciuti, P. Valdastri, A. Menciassi, and P. Dario, "Robotic magnetic steering and locomotion of capsule endoscope for diagnostic and surgical endoluminal procedures," Robotica, vol. 28, no. Special Issue 02, pp. 199-207, 2010.

[16] W. Yang, C. Hu, M. Li, M.-H. Meng, and S. Song, "A new tracking system for three magnetic objectives," IEEE Trans. Magn., vol. 46, no. 12, pp. 4023-4029, Dec. 2010.
[17] M. Li, S. Song, C. Hu, D. Chen, and M.-H. Meng, "A novel method of 6-DOF electromagnetic navigation system for surgical robot," in Proc. World Congr. Intell. Control Autom., Jul. 2010, pp. 2163-2167.

[18] T. Nara, H. Watanabe, and W. Ito, "Divergence form of Euler's equation for magnetic dipole localization," in Proc. SICE Annu. Conf., Sep. 2011, pp. 2356-2360.

[19] W. Fang and H. Son, "Optimization of measuring magnetic fields for position and orientation tracking," IEEE/ASME Trans. Mechatron., vol. 16, no. 3, pp. 440-448, Jun. 2011.

[20] M. Salerno, G. Ciuti, G. Lucarini, R. Rizzo, P. Valdastri, A. Menciassi, A. Landi, and P. Dario, "A discrete-time localization method for capsule endoscopy based on on-board magnetic sensing," Meas. Sci. Technol., vol. 23, no. 1, 2012, 015701.

[21] T. W. R. Fountain, P. V. Kailat, and J. J. Abbott, "Wireless control of magnetic helical microrobots using a rotating-permanent-magnet manipulator," in Proc. IEEE Int. Conf. Robot. Autom., 2010, pp. 576-581.

[22] B. J. Nelson, I. K. Kaliakatsos, and J. J. Abbott, "Microrobots for minimally invasive medicine," Аnпu. Rev. Biomed. Eng., vol. 12, no. 1, pp. 55-85, 2010.

[23] A. W. Mahoney and J. J. Abbott, "Managing magnetic force applied to a magnetic device by a rotating dipole field," Appl. Phys. Lett., vol. 99, 2011, 134103.

[24] A. W. Mahoney, D. L. Cowan, K. M. Miller, and J. J. Abbott, "Control of untethered magnetically actuated tools using a rotating permanent magnet in any position," in Proc. IEEE Int. Conf. Robot. Autom., 2012, pp. $3375-3380$.

[25] M. Beleggia, M. D. Graef, Y. T. Millev, D. A. Goode, and G. Rowlands, "Demagnetization factors for elliptic cylinders," J. Phys. D, Appl. Phys., vol. 38, pp. 3333-3342, 2005.

[26] J. A. Osborn, "Demagnetizing factors of the general ellipsoid," Phys. Rev., vol. 67, pp. 351-357, Jun. 1945.

[27] P. Rhodes and G. Rowlands, "Demagnetising energies of uniformly magnetised rectangular blocks," Proc. Leeds Phil. Liter. Soc., vol. 6, pp. 191-210, 1954.

[28] A. Aharoni, "Demagnetizing factors for rectangular ferromagnetic prisms," J. Appl. Phys., vol. 86, pp. 3432-3434, 1998.

[29] J. D. Jackson, Classical Electrodynamics. New York: Wiley, 1975.

[30] D. J. Griffiths, Introduction to Electrodynamics. Englewood Cliffs, NJ: Prentice-Hall, 1999. 